April 4, 1994 Revised: Jan 24, 1995

LBL-35452

UCB-PTH-94/08

\title{
q - deformed Dirac Monopole With Arbitrary Charge
}

\author{
Chong-Sun Chu, Pei-Ming Ho and Harold Steinackerf \\ Department of Physics \\ University of California \\ and \\ Theoretical Physics Group \\ Lawrence Berkeley Laboratory \\ University of California \\ Berkeley, CA 94720
}

\begin{abstract}
We construct the deformed Dirac monopole on the quantum sphere for arbitrary charge using two different methods and show that it is a quantum principal bundle in the sense of Brzezinski and Majid. We also give a connection and calculate the analog of its Chern number by integrating the curvature over $S_{q}^{2}$.
\end{abstract}

*email: hsteinac@physics.berkeley.edu 


\section{Introduction}

A major step towards a q - deformed gauge theory is to find a suitable concept of "quantum" fiber bundles. Recently some versions of quantum bundles have been proposed [1, 3], 田, where both the base space and the fiber are quantum spaces. While all of them have their particular advantages, only [1] also give a detailed concept of a connection on a principal fiber bundle. As examples, they construct explicitely the $\mathrm{q}$ - deformed Dirac Monopole for charge 1 and 2 , which are just $S U_{q}(2)$ resp. $S O_{q}(3)$. However, this construction is only formal for charge 1 and cannot be generalized to higher charges.

In this paper, we use the definition of [1] and give 2 explicit constructions of the deformed Dirac Monopole $P^{(n)}$ for arbitrary "integer" charge $[n]$ with connection in the sense of [1], both with universal and a general calculus inherited from the $3-\mathrm{D}$ calculus on $S U_{q}(2)$. We first find $P^{(n)}$ as a certain subalgebra of $S U_{q}(2)$, using a gradation on $S U_{q}(2)$. In the second approach, $P^{(2 n)}$ is constructed by "glueing together", in the classical spirit, 2 trivial bundles. We give a condition when this can be done in general. It is shown that the 2 constructions agree. While the bundles are defined for any integer $n$, we find local trivializations for even $n$ only; for odd $n$, they would only be formal. Thus we provide examples of nontrivial quantum principal bundles which are not quantum groups over some homogeneous space. In the 3-D calculus, analogs of Chern numbers are obtained by integrating the curvature two - form over the base $S_{q}^{2}$.

While the bundles are equipped with a star - structure, the trivializations respect this star - structure only in the classical limit (as in [1]). This appears to be a rather general feature of this approach to quantum bundles. While we take this as a feature rather than as a problem, it seems that more examples and results are needed to find the most fruitful definitions. Also, the connection one - forms are star - maps only in the classical limit. avoided if one wants to consider general gauge transformations. 


\section{The Dirac Monopole as a Subalgebra of $S U_{q}(2)$}

\subsection{Definition of quantum bundles}

Before giving the construction of the deformed monopole bundle, let us summarize the definition and main concepts of quantum principal bundles in the sense of [1], which we will use in this paper:

Definition 2.1 [1, Def 4.1] $P=P(B, A)$ is a quantum principal bundle (short: $Q P B$ ) with universal differential calculus, structure quantum group $A$ and base $B$ if

1. A is a Hopf algebra

2. $\left(P, \Delta_{R}\right)$ is a right $A$-comodule algebra; write $\Delta_{R}(p)=p^{1} \otimes p^{2} \in P \otimes A$

3. $B=P^{A}=\left\{u \in P: \Delta_{R} u=u \otimes 1\right\}$

4. $(\cdot \otimes \mathrm{id})\left(\mathrm{id} \otimes \Delta_{\mathrm{R}}\right): \mathrm{P} \otimes \mathrm{P} \rightarrow \mathrm{P} \otimes \mathrm{A}$ is a surjection (freeness condition)

5. ker $^{\sim}=\Gamma_{\text {hor }}$ (exactness condition for the differential envelope)

where horizontal forms $\Gamma_{h o r}$ are defined by

$$
\Gamma_{\text {hor }}=P\left(\Gamma_{B}\right) P \subseteq \Gamma_{P}
$$

and satisfy $\sim\left(\Gamma_{h o r}\right)=0$ identically. The left $P$ - module map $\sim$ is defined as

$$
\sim=\left.(\cdot \otimes i d) \circ\left(i d \otimes \Delta_{R}\right)\right|_{P^{2}}: \Gamma_{P} \rightarrow P \otimes \operatorname{ker} \epsilon .
$$

In the dual picture, it generates the fundamental (vertical) vector fields on the bundle. We will use the same symbol $\sim$ for the extended map in condition 4. .

A connection on a quantum principal bundle is an assignment of a left $P$-submodule $\Gamma_{\text {ver }} \subseteq \Gamma_{P}$ such that: 
1. $\Gamma_{P}=\Gamma_{h o r} \oplus \Gamma_{v e r}$,

2. projection $\Pi: \Gamma_{P} \rightarrow \Gamma_{v e r}$ is right invariant i.e.

$$
\Delta_{R} \Pi=(\Pi \otimes i d) \Delta_{R}
$$

A connection in $P$ is characterized by a right - invariant left $P$ - module map $\sigma: P \otimes \operatorname{ker} \epsilon \rightarrow \Gamma_{P}$ splitting the exact sequence

$$
0 \rightarrow \Gamma_{\text {hor }} \rightarrow \Gamma_{P} \stackrel{\sim}{\rightarrow} P \otimes \operatorname{ker} \epsilon \rightarrow 0,
$$

i.e. ${ }^{\sim} \circ \sigma=i d$. The connection form $\omega: A \rightarrow \Gamma_{P}$ is then given by

$$
\omega(a)=\sigma(1 \otimes(a-\epsilon(a))) .
$$

Conversely, $\sigma(p \otimes a)=p \omega(a)$ for $p \otimes a \in P \otimes \operatorname{ker} \epsilon$.

In order to use a general calculus, the above definitions have to be augmented. As usual, the first - order calculus on $A$ shall be determined by a right ideal $M_{A} \subset \operatorname{ker} \epsilon$ as $\Gamma_{A}=A^{2} / N_{A}$, where $N_{A}=\kappa\left(A \otimes M_{A}\right)$ and the map $\kappa: A \otimes A \rightarrow A \otimes A$ is given by

$$
\kappa\left(a \otimes a^{\prime}\right)=\sum a S a_{(1)}^{\prime} \otimes a_{(2)}^{\prime},
$$

where the Sweedler's notation [7] is employed.

Similarly on $P$, one assumes that the first - order differential structure $\Gamma_{P}$ is a quotient of the universal one, $\Gamma_{P}=P^{2} / N_{P}$ where $N_{P}$ is a sub-bimodule of $P^{2}$. The calculus for higher orders is then uniquely determined. In order to have consistent calculi on $P$ and $A$, the definition of a QPB is:

Definition 2.2 [1, Def 4.9] $P=P\left(B, A, N_{P}, M_{A}\right)$ is a quantum principal bundle with structure quantum group $A$, base $B$ and quantum differential calculi defined by $N_{P}, M_{A}$ if

1. A is a Hopf algebra 
2. $\left(P, \Delta_{R}\right)$ is a right $A$ - comodule algebra

3. $B=P^{A}=\left\{u \in P: \Delta_{R} u=u \otimes 1\right\}$

4. $(\cdot \otimes \mathrm{id})\left(\mathrm{id} \otimes \Delta_{\mathrm{R}}\right): \mathrm{P} \otimes \mathrm{P} \rightarrow \mathrm{P} \otimes \mathrm{A}$ is a surjection (freeness condition)

5. $\Delta_{R} N_{P} \subset N_{P} \otimes A$ (right covariance of differential structure).

6. $\sim\left(N_{P}\right) \subset P \otimes M_{A}$ (fundamental vector fields compatibility condition)

7. $\operatorname{ker}^{\sim}{ }_{N_{P}}=\Gamma_{\text {hor }}$ (exactness condition).

where $\sim_{N_{P}}$ is the map induced by $\sim$. A connection on a QPB with general calculus is again determined by a splitting $\sigma$ of the sequence

$$
0 \rightarrow \Gamma_{h o r} \rightarrow \Gamma_{P} \stackrel{\sim}{\stackrel{N_{P}}{\rightarrow}} P \otimes \operatorname{ker} \epsilon / M_{A} \rightarrow 0
$$

Point (6) may be replaced by the slightly stronger condition [1]

6.' $\quad \sim\left(N_{P}\right)=P \otimes M_{A}$, which we will adopt in section (3)ff.

\subsection{Dirac monopole with universal calculus}

$S U_{q}(2)$ is generated as usual by $\alpha, \beta, \gamma, \delta$ with the commutation relations

$$
\begin{gathered}
\alpha \beta=q \beta \alpha, \quad \alpha \gamma=q \gamma \alpha . \quad \alpha \delta=\delta \alpha+\left(q-q^{-1}\right) \beta \gamma \\
\beta \gamma=\gamma \beta, \quad \beta \delta=q \delta \beta, \quad \gamma \delta=q \delta \gamma
\end{gathered}
$$

and a determinant relation $\alpha \delta-q \beta \gamma=1$. The $*$-structure is $\alpha^{*}=\delta, \beta^{*}=$ $-q \gamma$.

It is shown in [1] that $P^{(1)}=S U_{q}(2)$ and $P^{(2)}=S O_{q}(3)$ are QPB 's with structure quantum group $A=k<Z^{1 / 2}, Z^{-1 / 2}>=U(1)$ resp. $A=k<$ $Z, Z^{-1}>=U(1)(k=\mathbb{C}$ in order to recover the classical monopole for $q=1)$, right coaction $\Delta_{R}: P \rightarrow P \otimes A$ defined by

$$
\Delta_{R}\left(\begin{array}{ll}
\alpha & \beta \\
\gamma & \delta
\end{array}\right)=\left(\begin{array}{ll}
\alpha \otimes Z^{1 / 2} & \beta \otimes Z^{-1 / 2} \\
\gamma \otimes Z^{1 / 2} & \delta \otimes Z^{-1 / 2}
\end{array}\right)
$$


and base $B=S U_{q}(2)^{A}=<1, b_{-}=\alpha \beta, b_{+}=\gamma \delta, b_{3}=\alpha \delta>S O_{q}(3)$ is defined here as the even elements of $S U_{q}(2)$.

To describe Dirac monopoles with higher charges, we define a degree for a monomial in $S U_{q}(2)$ as follows:

$$
\operatorname{deg}\left(\alpha^{a} \beta^{b} \gamma^{c} \delta^{d}\right)=a+c-b-d
$$

irrespective of ordering. We shall show that the q-deformed Dirac monopole with (classical) charge $n \in \mathbb{N}$ is

$$
P^{(n)}=<\left\{p \in S U_{q}(2), \operatorname{deg}(p)=n k, k \in \mathbb{Z}\right\}>
$$

and $A^{(n)}=k<Z^{n / 2}, Z^{-n / 2}>$. The superscript denotes the charge of the bundle. So monomials in $P^{(n)}$ have a degree which is an arbitrary multiple of $n$. For $n=2$ we have $P^{(2)}=S O_{q}(3)$, in agreement with [1]. The coaction $\Delta_{R}: P^{(n)} \rightarrow P^{(n)} \otimes A^{(n)}$ is induced from the above as a star - algebra map and is compatible with the grading, and $B=P^{(n) A}=<\left\{p \in P^{(n)}, \operatorname{deg}(p)=\right.$ $0\}>=S_{q}^{2}$. Also note that the above star - structure carries over to $P^{(n)}$.

We do not have to introduce trivializations and "local" bundles here, this will be done in the second approach. However to motivate the above definitions, let us remark that e.g. $\Phi_{0}\left(Z^{ \pm n / 2}\right)=\left(\delta^{-1} \alpha\right)^{ \pm n / 2}$ will turn out to be a local trivialization (at least for $q=1$ ); since locally $P=B \otimes A$, this gives the characterization above. In the classical limit, $A^{(n)}$ is nothing but the functions on $U(1)$ with $n$-th roots of unity identified, so the winding number of $P^{(n)}$ will just be $n$.

For $n \geq 2$, we can now show the following:

Proposition 2.3 $P^{(n)}\left(B, A^{(n)}\right)$ is a $Q P B$ with universal calculus.

\section{Proof}

For condition 5. of definition 2.1, we see that $\operatorname{ker}^{\sim}=\Gamma_{h o r}^{(1)} \cap \Gamma_{P}^{(n)}=\Gamma_{h o r}^{(n)}$, since $\operatorname{deg}(B)=0$. 
To show 4., consider the monomial $p \otimes Z^{n k / 2} \in P^{(n)} \otimes A^{(n)} \subset P^{(1)} \otimes A^{(1)}$ for $k \in \mathbb{Z}$. Since $P^{(1)}$ is a $\mathrm{QPB}$, there exists $\sum p_{i_{1}} \otimes p_{i_{2}} \in P^{(1)} \otimes P^{(1)}$ such that $\sim\left(\sum p_{i_{1}} \otimes p_{i_{2}}\right)=p \otimes Z^{n k / 2}$. Now, $\sim\left(\sum p_{i_{1}} \otimes p_{i_{2}}\right)=\sum p_{i_{1}} p_{i_{2}} \otimes Z^{\operatorname{deg}\left(p_{i_{2}}\right) / 2}$. Therefore, $\operatorname{deg}\left(p_{i_{2}}\right)=n k$, and $p_{i_{2}} \in P^{(n)}$ for all $i_{2}$. Also, $\operatorname{deg}\left(p_{i_{1}} p_{i_{2}}\right)=\operatorname{deg}(p)$ and $p \in P^{(n)}$, so $\operatorname{deg}\left(p_{i_{1}}\right) \in n \mathbb{Z}$. Hence, $p_{i_{1}} \in P^{(n)}$ for all $i_{1}$. Surjectivity is proved.

A possible connection one - form on $P^{(n)}$ is given by

$$
\begin{gathered}
\omega\left(Z^{k n / 2}\right)=S\left(\left(\alpha^{k n}\right)_{(1)}\right) d\left(\alpha^{k n}\right)_{(2)}=\kappa\left(1 \otimes\left(\alpha^{k n}-1\right)\right), \\
\omega\left(Z^{-k n / 2}\right)=S\left(\left(\delta^{k n}\right)_{(1)}\right) d\left(\delta^{k n}\right)_{(2)}=\kappa\left(1 \otimes\left(\delta^{k n}-1\right)\right)
\end{gathered}
$$

for $k>0$, where $\kappa$ is defined in [1]. This $\omega$ is well - defined, since $S\left(\left(\alpha^{k n}\right)_{(1)}\right)$, $S\left(\left(\delta^{k n}\right)_{(1)}\right),\left(\alpha^{k n}\right)_{(2)},\left(\delta^{k n}\right)_{(2)} \in P^{(n)}$. This connection was found observing that this is the trivial connection [1] obtained form the trivialization $\Phi\left(Z^{n / 2}\right)=\alpha^{n}$, which is a gauge - transformation of the trivialization (28) used in section 4 . Note that the above trivialization does not respect the star - structure even for $q=1$, nevertheless it is useful to e.g. find a connection; in the 3D - calculus, it simplifies to (18), and for even $n$, we would have obtained the same $\omega$ using (28). Quite generally, gauge - transformations tend to spoil the star - structure (and algebra - structure, as pointed out in [四]) of a trivialization.

To prove that $\omega$ defines a connection, we use Proposition 4.4 in [1]. We have to show

1. $\omega(1)=0$

2. $\sim \omega(a)=1 \otimes a-1 \otimes 1 \epsilon(a)$ for all $a \in A$

3. $\Delta_{R} \circ \omega=(\omega \otimes \mathrm{id}) \circ \operatorname{Ad}_{\mathrm{R}}$

1. is obvious, 2. holds since for $k>0$,

$$
\sim \omega\left(Z^{k n / 2}\right)=S\left(\left(\alpha^{k n / 2}\right)_{(1)}\right)\left(\alpha^{k n / 2}\right)_{(2)}^{(1)} \otimes\left(\alpha^{k n / 2}\right)_{(2)}^{(2)}-1 \otimes 1
$$




$$
\begin{aligned}
& =S\left(\left(\alpha^{k n / 2}\right)_{(1)}\right)\left(\alpha^{k n / 2}\right)_{(2)} \otimes Z^{k n / 2}-1 \otimes 1 \\
& =1 \otimes\left(Z^{k n / 2}-1\right)
\end{aligned}
$$

and similarly for $k<0$ as is easily seen from our coaction. For 3.,

$$
\begin{aligned}
\Delta_{R} \omega\left(Z^{k n / 2}\right) & =S\left(\left(\alpha^{k n / 2}\right)_{(1)}\right) d\left(\alpha^{k n / 2}\right)_{(2)} \otimes Z^{-k n / 2} Z^{k n / 2} \\
& =\omega\left(Z^{k n / 2}\right) \otimes 1=(\omega \otimes i d) A d_{R}\left(Z^{k n / 2}\right)
\end{aligned}
$$

and similarly for $k<0$.

\subsection{General Calculus}

It is also shown in [1] that $P^{(1)}\left(B, A^{(1)}, N_{P}^{(1)}, M_{A}^{(1)}\right)$ is a QPB with general calculus where $N_{P}^{(1)}$ defines the 3 -D calculus on $S U_{q}(2)$, i.e. the right ideal $M_{P}^{(1)}$ is generated by the six elements

$$
\delta+q^{2} \alpha-\left(1+q^{2}\right), \gamma^{2}, \beta \gamma, \beta^{2},(\alpha-1) \gamma,(\alpha-1) \beta
$$

and

$$
M_{A}^{(1)}=\pi\left(M_{P}^{(1)}\right)=<\left\{Z^{-1 / 2}+q^{2} Z^{1 / 2}-\left(1+q^{2}\right)\right\}>
$$

The projection $\pi: P \rightarrow A$ (dual of $U(1) \subset S U(2))$ is an algebra map

$$
\pi\left(\begin{array}{ll}
\alpha & \beta \\
\gamma & \delta
\end{array}\right)=\left(\begin{array}{ll}
Z^{1 / 2} & 0 \\
0 & Z^{-1 / 2}
\end{array}\right)
$$

For the general case, take $N_{P}^{(n)}=N_{P}^{(1)} \cap\left(P^{(n)}\right)^{2}, M_{A}^{(n)}$ generated by $Z^{-n / 2}+q^{2 n} Z^{n / 2}-\left(1+q^{2 n}\right)$, i.e. $Z^{n / 2} d Z^{n / 2}=q^{2 n} d Z^{n / 2} Z^{n / 2}$ or equivalently $N_{A}^{(n)}=N_{A}^{(1)} \cap\left(A^{(n)}\right)^{2}$. Then we have:

Proposition 2.4 $P^{(n)}\left(B, A^{(n)}, N_{P}^{(n)}, M_{A}^{(n)}\right)$ is a $Q P B$. 
Proof Conditions 1-4 of definition 2.2 are trivial as $P^{(n)}\left(B, A^{(n)}\right)$ is a QPB. Condition 5 is obvious because of our simple $\Delta_{R}$. Now, notice that $P^{(1)}\left(B, A^{(1)}, N_{P}^{(1)}, M_{A}^{(1)}\right)$ is a QPB, so $\operatorname{ker}^{\sim}{ }_{N_{P}^{(n)}}=\left(\operatorname{ker}^{\sim}{ }_{N_{P}^{(1)}}\right) \cap \Gamma_{P}^{(n)}=\Gamma_{\text {hor }}^{(1)} \cap$ $\left.\Gamma_{P}^{(n)} \cap \Gamma_{P}^{(n)}\right)=\Gamma_{h o r}^{(n)}$ since $\operatorname{deg}(\mathrm{B})=0$. For the same reason, we know that $\sim N_{P}^{(1)} \subset P^{(1)} \otimes M_{A}^{(1)}$. Therefore, $\sim N_{P}^{(n)} \subset P^{(1)} \otimes M_{A}^{(1)}$. But $\sim\left(p_{1} d p_{2}\right)=$ $p_{1} p_{2}^{(1)} \otimes p_{2}^{(2)}-p_{1} p_{2} \otimes 1 \in P^{(n)} \otimes A^{(n)}$, for all $p_{1}, p_{2} \in P^{(n)}$, so $\sim N_{P}^{(n)} \subset P^{(n)} \otimes M_{A}^{(n)}$. Hence we see that $P^{(n)}\left(B, A^{(n)}, N_{P}^{(n)}, M_{A}^{(n)}\right)$ is a QPB. Also, note that the $3 \mathrm{D}$ - calculus respects the star - structure [10].

The above connection one - form $\omega$ defines also a connection on $P^{(n)}$ with our general calculus. The only thing that remains to be checked according to Proposition 4.10 of [1] is $\omega\left(M_{A}^{(n)}\right)=0$. But this is clear since $\omega\left(Z^{-n / 2}+\right.$ $\left.q^{2 n} Z^{n / 2}-\left(1+q^{2 n}\right)\right)=\kappa\left(1 \otimes\left(\delta^{n}+q^{2 n} \alpha^{n}-\left(1+q^{2 n}\right)\right)\right) \in \kappa\left(1 \otimes M_{P}^{(n)}\right)$.

Thus, $\omega$ is a connection form on our bundle and is given by

$$
\begin{aligned}
\omega\left(Z^{k n / 2}\right) & =[k n]_{q^{-2} \omega^{1}}, \\
\omega\left(Z^{-k n / 2}\right) & =-[-k n]_{q^{-2}} \omega^{1}=-q^{2 k n} \omega\left(Z^{k n / 2}\right)
\end{aligned}
$$

if viewed in $S U_{q}(2)$, where $[n]_{q}=\frac{q^{n}-1}{q-1}$. This generalizes the result of [1] for $n=1$ and 2. Since $\left(\omega^{1}\right)^{*}=-\omega^{1}, \omega$ is a star - map for $q=1$ only. We have used $\omega^{1} \alpha=q^{-2} \alpha \omega^{1}, \omega^{1} \gamma=q^{-2} \gamma \omega^{1}$, where $\omega^{1}=\delta d \alpha-q^{-1} \beta d \gamma$ is a left invariant form in $S U_{q}(2)$.

To our knowledge, (10) is also a new description of the classical Dirac monopole.

\section{Combining patches to bundles}

Let us now show how nontrivial QPB's can be obtained by "glueing" together "local" bundles. To avoid repeating ourselves too much, we will give the following statements for the case of a general calculus only; the universal calculus is recovered by putting $M_{A}=N_{A}=N_{P}=0$. We first observe that 
the conditions 4 . and 7 . in definition 2.2 are equivalent to the exactness of the sequence $(\overline{0}) . \Gamma_{P}$.

Lemma 3.1 $P\left(B, A, N_{P}, M_{A}\right)$ satisfying conditions 1. to 3.,5. and 6.' of the definition 2.9 is a $Q P B$ with general calculus if and only if the sequence (1) is exact.

Proof Exactness of (7) at $\Gamma_{P}$ is just the condition 7. above.

Assume first $P$ is a QPB. Then by condition 4 . , for any $p \otimes a \in$ $P \otimes \operatorname{ker} \epsilon / M_{A}$ there exists $p_{1} \otimes p_{2} \in P \otimes P$ with $\sim\left(p_{1} \otimes p_{2}\right)=p_{1} p_{2}^{1} \otimes p_{2}^{2}=$ $p \otimes a$. Applying id $\otimes \epsilon$ to this equation we get $0=p_{1} p_{2}^{1} \epsilon\left(p_{2}^{2}\right)=p_{1} p_{2}$, i.e. $p_{1} \otimes p_{2} \in \Gamma_{P}$, which shows that ${ }^{\sim}$ in (7) is surjective, so it is exact.

Conversely, suppose (7) is exact. Take any $p \otimes a=p \otimes(a-\epsilon(a))+$ $p \otimes \epsilon(a) \in P \otimes A$. Since $\sim_{N_{P}}$ is surjective, there exists $p_{1} d p_{2} \in \Gamma_{P}$ with $\sim_{N_{P}}\left(p_{1} d p_{2}\right)=p \otimes(a-\epsilon(a))+P \otimes M_{A}$. Now $\sim(p \otimes \epsilon(a))=p \otimes \epsilon(a)$ and from 6'. $\sim\left(N_{P}\right)=P \otimes M_{A}$, so condition 4 . is satisfied.

Assume now we have 3 quantum principal bundles $P_{0}\left(B_{0}, A, N_{0}, M_{A}\right)$, $P_{1}\left(B_{1}, A, N_{1}, M_{A}\right) \subset P_{01}\left(B_{01}, A, N_{01}, M_{A}\right)$ ( $P_{01}$ corresponds to the bundle on the "overlap" $B_{01}$ of $B_{0}$ and $\left.B_{1}\right)$ and we would like to know if $P_{0}$ and $P_{1}$ can be understood as two patches of a "global" quantum bundle $P\left(B, A, N, M_{A}\right) \subset$ $P_{0}, P_{1} \subset P_{01}$. A natural guess is that $P=P_{0} \cap P_{1}$. In this case the coactions $\Delta_{R_{i}}: P_{i} \rightarrow P_{i} \otimes A$ certainly must agree in $P$. If we want a connection on $P$, then we should also have connection forms $\omega_{i}: A \rightarrow \Gamma_{P_{i}}$ which agree on the overlap, i.e. $\omega_{0}(a)=\omega_{1}(a)$ in $\Gamma_{P_{01}}$.

However, some care must be taken if we want to compare differential forms on different patches. First of all, the differential structures on $P_{i}$ must be compatible, i.e. we should have $N_{0}=N_{01} \cap P_{0}^{2}, N_{1}=N_{01} \cap P_{1}^{2}$ and $N_{P} \equiv N=N_{01} \cap P^{2}=N_{0} \cap N_{1}$. But this is not enough: Suppose we have any 2 differential forms - not necessarily connections $-\omega_{0} \in \Gamma_{P_{0}}$ and $\omega_{1} \in \Gamma_{P_{1}}$ and find by doing calculations in $\Gamma_{P_{01}}$ that they are equal. One would certainly like to conclude, as in the classical case, that they determine a 
"global" form $\omega$ in $\Gamma_{P}$. This is not evident, it is a condition on the calculus. It motivates the following definition: The above calculi on $P_{0}, P_{1}, P_{01}$ are called admissible if

$$
\omega_{0}=\omega_{1}+n_{01} \quad \text { for } \quad \omega_{i} \in \Gamma_{P_{i}}
$$

implies that there exists a $\omega \in \Gamma_{P}$ such that

$$
\omega=\omega_{0}+n_{0}=\omega_{1}+n_{1}, \quad n_{i} \in N_{i} .
$$

In other words, $\omega_{0}=\omega_{1}$ determines a $\omega \in \Gamma_{P_{0}} \cap \Gamma_{P_{1}}=\Gamma_{P}$, where the intersection is defined as intersection of the cosets.

A calculus which does not satisfy this condition would be highly unpracticable for global statements. The universal calculus is certainly admissible since $\left(P_{0} \otimes P_{0}\right) \cap\left(P_{1} \otimes P_{1}\right)=P \otimes P$ implies $\Gamma_{P_{0}} \cap \Gamma_{P_{1}}=\Gamma_{P}$. The calculus we will consider on the monopole - bundle will be shown to be admissible too, using a fairly general line of reasoning.

Theorem 3.2 In the above situation, $P=P_{0} \cap P_{1}=P\left(B, A, N, M_{A}\right)$ is a quantum principal bundle with base $B=B_{0} \cap B_{1}$ and connection if we have admissible differential structures which satisfy $\sim(N)=P \otimes M_{A}$, connection forms $\omega_{0}=\omega_{1}$ on $P_{0}$ resp. $P_{1}$, and $\Gamma_{0 h o r} \cap \Gamma_{1 h o r}=\Gamma_{h o r}$. Conversely, if $P=P_{0} \cap P_{1}$ is a quantum principal bundle, then $\Gamma_{0 h o r} \cap \Gamma_{1 h o r}=\Gamma_{\text {hor }}$.

Proof First, $\Delta_{R}(p) \in P_{0} \otimes A \cap P_{1} \otimes A=P \otimes A$ for $p \in P$ implies 2. in Def 2.1. Further, $B=P^{A}=\left(P_{0} \cap P_{1}\right)^{A}=P_{0}^{A} \cap P_{1}^{A}=B_{0} \cap B_{1}$. By the above definition of the differential structures condition 5 . is satisfied, since $\Delta_{R_{i}}: P_{i} \rightarrow P_{i} \otimes A$ do not "leave" the bundles.

Assume $\Gamma_{0 h o r} \cap \Gamma_{1 h o r}=\Gamma_{\text {hor }}$. Since $\omega_{0}(a)=\omega_{1}(a)$ and the calculus is admissible, this defines $\omega(a) \in \Gamma_{P}$ and $\sigma(p \otimes a)=p \omega(a) \in \Gamma_{P}$ for $(p \otimes a) \in$ $P \otimes \operatorname{ker} \epsilon$. From proposition 4.10 in [1] it follows that $\omega$ is a connection $1-$ form. Now $\sim(p \otimes a)=p \otimes a$ shows that the map $\sim$ in (7) is surjective.

It remains to show $\mathrm{ker}^{\sim}=\Gamma_{\text {hor }}$. Let $p_{1} d p_{2} \in \Gamma_{P}$. Since $P_{0}$ and $P_{1}$ are quantum bundles, $\sim\left(p_{1} d p_{2}\right)=0$ implies $p_{1} d p_{2} \in \Gamma_{\text {ohor }} \cap \Gamma_{1 h o r}=\Gamma_{\text {hor }}$ by 
assumption. Now Lemma 3.1 tells us that $P\left(B, A, N, M_{A}\right)$ is a quantum principal bundle.

Conversely, assume $P=P_{0} \cap P_{1}$ is a quantum principal bundle. Let $p_{1} d p_{2} \in \Gamma_{0 h o r} \cap \Gamma_{1 h o r}$. Then $\sim\left(p_{1} d p_{2}\right)=0$. Since $\Gamma_{0 h o r} \cap \Gamma_{1 h o r} \subset \Gamma_{P_{0}} \cap \Gamma_{P_{1}}=\Gamma_{P}$ and $P$ is a QPB, this implies $p_{1} d p_{2} \in \Gamma_{\text {hor }}$. The other inclusion $\Gamma_{0 h o r} \cap \Gamma_{1 h o r} \supset$ $\Gamma_{h o r}$ is trivial.

explicitely. If there are several "patches" $P_{i}$, then the above theorem generalizes inductively in an obvious way. One can show that if $\sim\left(N_{P}\right)=$ $P \otimes M_{A}$, then $\Gamma_{0 h o r} \cap \Gamma_{1 h o r}=\Gamma_{h o r}$ follows from $\Gamma_{\text {ohor }}^{u} \cap \Gamma_{1 h o r}^{u}=\Gamma_{h o r}^{u}$ (universal calculus). More generally, we have

Lemma 3.3 If $P(B, A)$ is a $Q P B$ with universal calculus and we have $N_{P}$ and $M_{A}$ satisfying conditions 5. and 6'. of definition 2.9, then $P\left(B, A, N_{P}, M_{A}\right)$ is a $Q P B$ with general calculus. Conversely, if $P\left(B, A, N_{P}, M_{A}\right)$ is a $Q P B$ and $\sim(n)=0$ for $n \in N_{P}$ implies $n \in \Gamma_{\text {hor }}^{u}$, then $P(B, A)$ is a $Q P B$ with universal calculus.

Proof First suppose $P(B, A)$ is a QPB; we have to show that $\operatorname{ker}^{\sim}{ }_{N_{P}} \subset$ $\Gamma_{\text {hor }}$. Let $\sim_{N_{P}}(\gamma)=0$. This means $\sim(\gamma) \in P \otimes M_{A}=\sim\left(N_{P}\right)$ by 6 '. . So there is a $n \in N_{P}$ with $\sim(n-\gamma)=0$. But $P$ is a QPB with universal calculus, so it follows $\gamma \in \Gamma_{h o r}^{u}+n$, i.e. $\gamma \in \Gamma_{h o r}$.

The converse statement can be proved similarly.

\section{Dirac Monopoles by Patching Two Trivial QPBs}

We can now present the second construction of the Dirac monopoles for general calculus as an illustration of the general method above. This will be done for even "charge" only; for odd charge, the trivializations etc. would only be formal. 
We define two trivial QPBs $P_{0}^{(2 n)}$ and $P_{1}^{(2 n)}$, and then show that $P^{(2 n)}=$ $P_{0}^{(2 n)} \cap P_{1}^{(2 n)}$ is the monopole of charge $2 n$.

For $P_{0}^{(2 n)}$, as motivated by the charge 2 case in [1], we now try to define the base $B_{0}$, fiber $A^{(2 n)}$ and trivial bundle $P_{0}^{(2 n)}$ be specified by their generators as:

$$
\begin{aligned}
B_{0}= & <\left\{1, b_{-}, b_{+}, b_{3},\left(b_{3}+q^{2 m}-1\right)^{-1} ; \quad m \in \mathbb{Z}\right\}>, \\
A^{(2 n)}= & <\left\{Z^{n}, Z^{-n}\right\}>, \\
P_{0}^{(2 n)}= & <B_{0} \cup\left\{\left(\delta^{-1} \alpha\right)^{n},\left(\alpha^{-1} \delta\right)^{n}\right\}>.
\end{aligned}
$$

We are going to show that they give a trivial QPB.

The commutation relations between the generators of $P_{0}$ are induced by $S U_{q}(2)$ through the following expressions [6]:

$$
b_{-}=\alpha \beta, \quad b_{+}=\gamma \delta, \quad b_{3}=\alpha \delta,
$$

where $\alpha, \beta, \gamma, \delta$ are generators of $S U_{q}(2)$ with the well-known relations stated before. The commutation relations involving inverses are obtained by multiplying them from both sides by inverses of generators.

In the classical limit $q=1, B_{0}$ becomes the algebra of the functions on $S^{2} \backslash\left\{\right.$ south pole\}, and $b_{ \pm}= \pm(x \pm i y), b_{3}=z+1 / 2$, where $x, y, z$ are the Cartesian coordinates. Note that $\alpha \delta-\beta \gamma=1$ is equivalent to $x^{2}+y^{2}+z^{2}=$ $(1 / 2)^{2}$. The somewhat complicated definition here (see [2]) will become clear below. $P_{0}^{(2 n)}$ as a trivial bundle is generated by the base $B_{0}$ and the fibers, cp. (28).

Define a coaction $\triangle_{R}$ on $P_{0}^{(2 n)}$ such that $B_{0}=\left(P_{0}^{(2 n)}\right)^{A^{(2 n)}}$ :

$$
\begin{aligned}
\triangle_{R}(1) & =1 \otimes 1, \\
\triangle_{R}\left(b_{i}\right) & =b_{i} \otimes 1, \quad i=-,+, 3, \\
\triangle_{R}\left(\left(\delta^{-1} \alpha\right)^{ \pm n}\right) & =\left(\delta^{-1} \alpha\right)^{ \pm n} \otimes Z^{ \pm n} .
\end{aligned}
$$

The trivialization $\Phi_{0}$ is defined as

$$
\Phi_{0}(1)=1, \quad \Phi_{0}\left(Z^{ \pm n}\right)=\left(\delta^{-1} \alpha\right)^{ \pm n}
$$


which generalizes the trivialization in [1]. To see that we have a trivial QPB, we first have to show that $B_{0}$ is the invariant subalgebra of $P_{0}^{(2 n)}$ under the above coaction. This is clear if any $p_{0} \in P_{0}^{(2 n)}$ can be written as a sum of terms $B_{0}\left(\delta^{-1} \alpha\right)^{k n}$. Thus we must be able to commute $B_{0}$ through $\left(\delta^{-1} \alpha\right)$. Writing down the commutation relations explicitely, one can always obtain relations like $\alpha B_{0} \alpha^{-1} \in B_{0}$. Note that for $m \in \mathbb{Z}, q^{-2 m} \alpha^{-m} b_{3}^{-1} \alpha^{m}=$ $q^{-2 m} \delta^{m} b_{3}^{-1} \delta^{-m}=\left(b_{3}+q^{2 m}-1\right)^{-1}($ cp. [2] $)$ and so in general,

$$
q^{-4 n k}\left(\alpha^{-1} \delta\right)^{n k}\left(b_{3}+q^{2 m}-1\right)^{-1}\left(\delta^{-1} \alpha\right)^{n k}=\left(b_{3}+q^{4 n k+2 m}-1\right)^{-1}, \quad k \in \mathbb{Z} .
$$

This shows that $B_{0}$, as defined in (21) is the invariant subalgebra, and one can also see the necessity to include all the generators of $B_{0}$.

$\Phi_{0}$ is convolution-invertible with $\Phi_{0}^{-1}\left(Z^{ \pm n}\right)=\left(\delta^{-1} \alpha\right)^{\mp n}$, and is also an intertwiner: $\triangle_{R} \circ \Phi_{0}=\left(\Phi_{0} \otimes i d\right) \circ \triangle_{A}$, where $\triangle_{A}\left(Z^{n}\right)=Z^{n} \otimes Z^{n}$ is the coproduct on $A^{(2 n)}$. So $P_{0}^{(2 n)}$ is a trivial QPB.

Below we will need the following alternative representation of $P_{0}^{(2 n)}$ :

$$
P_{0}^{(2 n)}=\left\{p \in<S U_{q}(2) \cup\left\{(\delta \alpha)^{-1},(\alpha \delta)^{-1}\right\}>: \operatorname{deg}(p)=2 k n, k \in \mathbb{Z}\right\} \equiv \tilde{P}_{0}^{(2 n)},
$$

i.e. the algebra generated by $S U_{q}(2)$ and $(\delta \alpha)^{-1},(\alpha \delta)^{-1}$, with degrees being multiples of $2 n$. To see this, note that $P_{0}^{(2 n)} \subset \tilde{P}_{0}^{(2 n)}$ because $\delta^{-1} \alpha=(\alpha \delta)^{-1} \alpha^{2}$ etc. and $b_{3}^{-1}=(\alpha \delta)^{-1}$, so $\alpha^{-n} b_{3}^{-1} \alpha^{n} \in \tilde{P}_{0}^{(2 n)}$ also. To see the other inclusion, we first show that $B_{0}$ is also the invariant subalgebra (under the coaction of $\left.A^{(2 n)}\right)$ of $\tilde{P}_{0}^{(2 n)}$ : we have just seen $B_{0} \subset \tilde{P}_{0}^{(2 n)}$, and the same commutation relations as above show that indeed $B_{0}=\left(\tilde{P}_{0}^{(2 n)}\right)^{A^{(2 n)}}$. But this means that $\tilde{P}_{0}^{(2 n)}$ is a QPB with the same trivialization $\Phi_{0}$ as above. Thus we know (from [1] Example 4.2) that $\tilde{P}_{0}^{(2 n)}=B_{0} \Phi_{0}\left(A^{(2 n)}\right)=P_{0}^{(2 n)}$.

The discussion on $P_{1}^{(2 n)}$ is parallel to that on $P_{0}^{(2 n)}$, but much easier. Therefore we just give the relevant equations:

$$
\begin{aligned}
B_{1} & =<\left\{1, b_{-}, b_{+}, b_{3},\left(b_{3}-1\right)^{-1}\right\}>, \\
P_{1}^{(2 n)} & =<B_{1} \cup\left\{\left(\gamma \beta^{-1}\right)^{n},\left(\beta \gamma^{-1}\right)^{n}\right\}>,
\end{aligned}
$$




$$
\begin{aligned}
A^{(2 n)} & =<\left\{Z^{n}, Z^{-n}\right\}>, \\
\triangle_{R}\left(b_{i}\right) & =b_{i} \otimes 1, \quad i=-,+, 3, \\
\triangle_{R}\left(\left(\gamma \beta^{-1}\right)^{ \pm n}\right) & =\left(\gamma \beta^{-1}\right)^{ \pm n} \otimes Z^{ \pm n} \\
\Phi_{1}\left(Z^{ \pm n}\right) & =\left(-\gamma \beta^{-1}\right)^{ \pm n}
\end{aligned}
$$

and $P_{1}^{(2 n)}$ is also a trivial QPB. Note again that $\operatorname{deg}\left(B_{1}\right)=0$ and $\operatorname{deg}\left(\Phi_{i}\left(Z^{n}\right)\right)=$ $2 n$.

The "overlap" $P_{01}^{(2 n)}$ of $P_{0}^{(2 n)}$ and $P_{1}^{(2 n)}$ is similarly defined by

$$
\begin{aligned}
B_{01} & =<B_{0} \cup\left\{\left(b_{3}-1\right)^{-1}\right\}>, \\
P_{01}^{(2 n)} & =<B_{01} \cup\left\{\left(\gamma \beta^{-1}\right)^{ \pm n},\left(\delta^{-1} \alpha\right)^{ \pm n}\right\}>
\end{aligned}
$$

and so on as above. On $P_{01}^{(2 n)}$, both trivializations can be used, with the transition function

$$
\gamma_{01}\left(Z^{n}\right)=\Phi_{0}\left(Z^{n}\right) \Phi_{1}^{-1}\left(Z^{n}\right)=\left(-q^{2} b_{3}^{-1} b_{-}^{2}\left(b_{3}-1\right)^{-1}\right)^{n} \in B_{01}
$$

It should be noted that while these trivial bundles are closed under the star - operation, the maps $\Phi_{i}$ respect this star - structure only for $q=1$. This appears to be very hard to avoid in this framework, and we accept it here.

Now define the Dirac - monopole bundle with charge $2 n$ by

$$
P^{(2 n)}=P_{0}^{(2 n)} \cap P_{1}^{(2 n)}
$$

We will now show that for even charges this construction agrees with the one in section 2. First, we prove

\section{Proposition 4.1}

$$
P^{(2 n)}=<p \in S U_{q}(2): \operatorname{deg}(p)=2 n k, k \in \mathbb{Z}>.
$$


Proof Let $p_{0}, p_{1} \in P_{0}^{(2 n)}$ resp. $P_{1}^{(2 n)}$ and $p_{0}=p_{1}$. Note that $\beta, \beta^{-1}$, $\gamma, \gamma^{-1}$ can be commuted through any terms by just picking up powers of $q$. Multiplying $\alpha^{-1}, \delta^{-1}$ to the relation $\alpha \delta=\delta \alpha+(q-1)\left(\frac{q+1}{q}\right) \beta \gamma$ appropriately from both sides, one gets relations like $\delta \alpha^{-1}=\alpha^{-1} \delta+(q-1)(\ldots)$ and $\alpha^{-1} \delta^{-1}=\delta^{-1} \alpha^{-1}+(q-1)(\ldots)$, i.e. one can order thing in any way up to terms proportional to $(q-1)$.

Let us define a normal form for $p_{1}$ as follows: bring all $\beta, \gamma$ to the right of all $\alpha, \delta$ and order $\alpha$ to the left of $\delta$, picking up terms proportional to $(q-1)$. Then replace all terms $\alpha \delta$ by $(1+q \beta \gamma)$. Putting $\gamma$ to the right of $\beta, p_{1}$ finally has the form either $\alpha^{n} \beta^{x} \gamma^{y}+(q-1)(\ldots), \delta^{n} \beta^{x} \gamma^{y}+(q-1)(\ldots)$ or $\beta^{x} \gamma^{y}+(q-1)(\ldots)$ with $x, y \in \mathbb{Z}, n \in N \mathrm{~N}$.

Similarly, define a normal form for $p_{0}$ as follows: bring all $\beta, \gamma$ to the right of all $\alpha, \delta$, order $\beta$ to the left of $\gamma$ and replace all terms $\beta \gamma$ by $(\alpha \delta-1) / q$. Now order $\alpha$ to the left of $\delta$ picking up terms prop. to $(q-1) . p_{0}$ finally has the form either $\alpha^{x} \delta^{y} \beta^{n}+(q-1)(\ldots), \alpha^{x} \delta^{y} \gamma^{n}+(q-1)(\ldots)$ or $\alpha^{x} \delta^{y}+(q-1)(\ldots)$ with $x, y \in \mathbb{Z}, n \in M \mathrm{~W}$. Now consider the equation

$$
p_{0}=p_{1} .
$$

and put terms in $p_{1}$ which do not contain inverses to the left side, in normal form for $p_{0}$ (only for monomials which are not proportional to $(q-1)$, say). Then let $q=1$ and consider both sides as classical functions on $S U(2)$. All terms proportional to $(q-1)$ vanish, and all remaining monomials are in normal form on both sides and are easily seen to be independent as functions on $S U(2)$. This implies that all coefficients are actually zero, i.e. all terms on both sides are proportional to $(q-1)$. (or simply: classical functions defined on both patches are defined globally on $S U(2))$. We can now cancel the greatest common power of $(q-1)$, put regular terms to the left and apply the same argument. This cannot go on forever since the right side can be ordered completely, so both sides must be zero eventually, proving that $p_{0}=p_{1} \in S U_{q}(2)$. Using (30), this immediately shows that

$$
P^{(2 n)}=<p \in S U_{q}(2): \operatorname{deg}(p)=2 n k, k \in \mathbb{Z}>,
$$


as claimed.

The essence of the proof is to write things in the form ("class" $)+(q-1)$ ("quantum") and to apply classical reasoning to ("class"), which should be a fairly general strategy. Proposition 4.1 and (30) generalize the result of [1] for $n=1$.

We can now introduce the same induced 3-D calculus on the bundles as in section 2 , i.e. the calculus on the patches $P_{i}^{(2 n)}$ is defined by

$$
N_{P_{i}}^{(2 n)}=P_{i}^{(2 n)} N_{P}^{(2 n)} P_{i}^{(2 n)}
$$

with the same ideals as in section 2. Using ${ }^{\sim}=(i d \otimes \pi) \kappa^{-1}$ in a Hopf algebra one can easily see $\sim\left(N_{P}^{(1)}\right)=P^{(1)} \otimes M_{A}^{(1)}$, and $\sim\left(N_{P}^{(2 n)}\right)=P^{(n)} \otimes M_{A}^{(2 n)}$ with a similar argument as in section 2. So $P_{i}^{(n)}$ are trivial QPB with this calculus by example (4.11) in [1].

It was already shown in section 2 that

$$
\begin{gathered}
\omega\left(Z^{k n}\right)=S\left(\left(\alpha^{2 k n}\right)_{(1)}\right) d\left(\alpha^{2 k n}\right)_{(2)}=\kappa\left(1 \otimes\left(\alpha^{2 k n}-1\right)\right), \\
\omega\left(Z^{-k n}\right)=S\left(\left(\delta^{2 k n}\right)_{(1)}\right) d\left(\delta^{2 k n}\right)_{(2)}=\kappa\left(1 \otimes\left(\delta^{2 k n}-1\right)\right)
\end{gathered}
$$

for $k \in \mathrm{N}$ defines a connection one - form. Any monomials of degree $2 n k$ in $P_{i}^{(2 n)}$ can be written in the form $\Phi_{i}\left(Z^{2 n k}\right) B$ or $B \Phi_{i}\left(Z^{2 n k}\right)$, and so one can put $\omega$ in the standard form of a connection one-form in $P_{0}^{(2 n)}$ and $P_{1}^{(2 n)}$ :

$$
\omega(a)=\Phi_{i}^{-1}(a) \beta_{i}(a) \Phi_{i}(a)+\Phi_{i}^{-1}(a) d \Phi_{i}(a) ; i=0,1 ; a \in A^{(2 n)},
$$

where $\beta_{i} \in \Gamma_{\text {ihor }}^{(2 n)}$ and $\beta_{i}(1)=0$.

Now let us show the following:

Proposition 4.2 The calculus on $P^{(2 n)}, P_{0}^{(2 n)}, P_{1}^{(2 n)}$ is admissible (defined by (19), (29)). 
Proof The reasoning is as in the previous proposition. Assume we have $\omega_{0}, \omega_{1}$ in $\Gamma_{P_{0}}^{(2 n)}$ resp. $\Gamma_{P_{1}}^{(2 n)}$ with $\omega_{0}=\omega_{1}$ in $\Gamma_{P_{01}}^{(2 n)}$. Since in the $3 \mathrm{D}$ - calculus all one - forms on $S U_{q}(2)$ and thus on $P_{i}^{(2 n)}$ can be written in terms of three left - invariant Maurer - Cartan forms $\omega^{0}, \omega^{1}, \omega^{2}$ which have simple commutation relations

$$
\begin{array}{ll}
\omega^{0} \alpha=q^{-1} \alpha \omega^{0}, & \omega^{0} \beta=q \beta \omega^{0}, \\
\omega^{1} \alpha=q^{-2} \alpha \omega^{1}, & \omega^{1} \beta=q^{2} \beta \omega^{1}, \\
\omega^{2} \alpha=q^{-1} \alpha \omega^{2}, & \omega^{2} \beta=q \beta \omega^{2} .
\end{array}
$$

and similarly with the inverses $\alpha^{-1}$ etc., we can commute the forms to the right and have $\omega_{0}=f_{k} \omega^{k}, \quad \omega_{1}=g_{k} \omega^{k}$ (summation implied), so

$$
f_{k} \omega^{k}=g_{k} \omega^{k} .
$$

As in proposition 4.1 put both $f_{k}$ and $g_{k}$ in their respective normal form ("class") $+(q-1)$ ("quant") and bring all regular terms of $g_{k}$ to the left side. Then putting $q=1$, the "classical" parts are all independent as one - forms since the $\omega_{i}$ are and therefore vanish. Cancelling $(q-1)$ and repeating the argument, it follows that $\omega_{0}$ and $\omega_{1}$ are elements of $\Gamma_{P}^{(1)}$ and in fact in $\Gamma_{P}^{(2 n)}$, since the degree is conserved.

Now we can use theorem 3.2: suppose $\rho \in \Gamma_{0 h o r}^{(2 n)} \cap \Gamma_{1 h o r}^{(2 n)}$, so $\rho \in \Gamma_{P}^{(2 n)}$. We can expand it as above

$$
\rho=f_{0} \omega^{0}+f_{1} \omega^{1}+f_{2} \omega^{2},
$$

with $f_{i} \in P^{(2 n)}$. But $\omega^{0}$ and $\omega^{2}$ are horizontal (explicitely: $\omega^{0}=\delta^{2} d b_{-}+$ $q^{-2} \beta^{2} d b_{+}-q^{-1}\left(1+q^{-2}\right) \beta \delta d b_{3}$ and $\omega^{2}=-\gamma^{2} d b_{-}-q^{-2} \alpha^{2} d b_{+}+q^{-1}\left(1+q^{-2}\right) \alpha \gamma d b_{3}$ ), while $\omega^{1}$ is not. Therefore $f_{1}=0$, and $\rho \in \Gamma_{\text {hor }}^{(2 n)}$, since all coefficients of $d B$ must have degree $2 n$. So $P^{(2 n)}$ is a QPB with a general differential calculus, with the same connection form $\omega$ restricted to elements $a \in A^{(2 n)}$.

Finally we would like to mention that since the trivializations are not "real" for $q \neq 1$, one might just go ahead and use trivializations such as 
$\Phi\left(Z^{1 / 2}\right)=\alpha$ which do not respect the star - structure even for $q=1$, at least as computational tools. Since we know that the "global" bundle with the star - structure does have the correct classical limit, this may be an acceptable and useful strategy, and deserves further consideration.

\section{Concluding Remarks}

\subsection{A note on gauge transformations}

A gauge transformation is a convolution invertible map $\gamma: A \rightarrow B$ :

$$
\gamma * \gamma^{-1}=\gamma^{-1} * \gamma=1
$$

Let us define the "primitive charge" of a monomial in $B$ as $\left(n_{-}-n_{+}\right)$, where $n_{ \pm}$are the total powers of $b_{ \pm}$appearing in the monomial or equivalently (power of $\alpha$ - power of $\delta$ ). This is preserved by the commutation relations, as our previous degree. Suppose that $\gamma=\sum_{k=i}^{j} \gamma^{(k)}$, where each $\gamma^{(k)}$ contains only monomials that have primitive charge $k$. Hence $i$ and $j$ are the minimum and maximum of the primitive charges of all monomials in $\gamma$. Let the convolution inverse of $\gamma$ be denoted in the same way: $\gamma^{-1}=\sum_{k=i \prime}^{j \prime} \gamma^{(k)}$ I. So

$$
1=\epsilon\left(Z^{n / 2}\right) \cdot 1=\gamma * \gamma^{-1}=\sum_{k=i+i \prime}^{j+j \prime} \gamma^{(k)} / \prime
$$

which implies that $i+i \prime=j+j \prime=0$. The only possibility that this can be true is that $i=j=-i \prime=-j \prime$, which means that all monomials in $\gamma$ have the same primitive charge $n$. However, this means in the classical limit that $\gamma$ is proportional to $e^{i n \phi}$. That is, by admitting only finite sums in a convolution invertible $\gamma$ one is restricting oneself to a very special, rigid class of gauge transformations. Thus infinite series cannot be avoided in general. 


\subsection{Remarks on the Chern Class}

Classically, the monopole charge $n$ is given by an integration over the base of the first Chern class

$$
\frac{1}{2 \pi i} \int_{S^{2}} F=n,
$$

where $F=d A_{+}=d A_{-}$. Here $A_{+}, A_{-}$are the connection form on the northern and southern hemisphere respectively and the global connection form is given in terms of trivalizations as

$$
\omega=\left\{\begin{array}{l}
A_{+}+i d \varphi_{+}, \text {on }_{+} \\
A_{-}+i d \varphi_{-}, \text {on } H_{-}
\end{array}\right.
$$

with $e^{i \varphi_{+,-}}$being the local trivalization.

In the deformed case, we have the global connection form $\omega$. Suppose it is written in terms of trivialization as [回]

$$
\omega=\phi_{i}^{-1} \beta_{i} \phi_{i}+\phi_{i}^{-1} d \phi_{i},
$$

then it is not hard to check that

$$
d \omega=\phi_{i}^{-1}\left(d \beta_{i}+\beta_{i} \beta_{i}\right) \phi_{i}=d \omega+\omega \omega,
$$

which is in fact the curvature 2 -form on $P$ ([5], cp. [1]). Carrying the $\phi_{i}$ through the $d \omega$, we get

$$
d \beta_{i}+\beta_{i} \beta_{i}=q^{2 n} d \omega=q^{2 n}[n]_{q^{-2}} d \omega^{1}
$$

which is again equal for the two patches and explicitely horizontal. It leads us to define the deformed Chern class as

$$
\frac{1}{2 \pi i} F=\frac{1}{2 \pi i}\left(d \beta_{i}+\beta_{i} \beta_{i}\right)=\frac{q^{2 n}}{2 \pi i}[n]_{q^{-2}} d \omega^{1} .
$$

Consider the base $B=S_{q}^{2}=<b_{+}, b_{-}, b_{3}>\subset S U_{q}(2)$, with the calculus inherited from the 3-D calculus on $S U_{q}(2)$. Denote $\Gamma_{B}=B d B$ and introduce 
the set $\Gamma_{B}^{\wedge^{2}}$ of 2 forms on $B$. Notice that $\Gamma_{B}^{\wedge^{2}}$ contains elements of the form $B d b_{i} d b_{j}, i, j=-,+, 3$. Since

$$
\begin{aligned}
& d b_{+}=\gamma^{2} \omega^{0}-q^{2} \delta^{2} \omega^{2}, \\
& d b_{-}=\alpha^{2} \omega^{0}-q^{2} \beta^{2} \omega^{2}, \\
& d b_{3}=\alpha \gamma \omega^{0}-q^{2} \beta \delta \omega^{2} .
\end{aligned}
$$

So, $\Gamma_{B}^{\wedge^{2}}=B \omega^{0} \omega^{2}=B d \omega^{1}$. Because $d \omega^{1}$ is a central element in $B_{01}$, under a gauge transformation $U \in B_{01}$ we have $F \rightarrow U^{-1} F U=F$.

Notice that $\omega^{0} \omega^{2}$ is manifestly left invariant under the coaction of $S U_{q}(2)$, and is the unique top 2 -form on $B$. This allow us to introduce a linear functional

$$
\begin{gathered}
\int_{B}: \Gamma_{B}^{\wedge^{2}} \rightarrow \mathbb{C} \\
\int_{S_{q}^{2}} a d \omega^{1}=2 \pi i<a>_{S U_{q}(2)}, \forall a \in B,
\end{gathered}
$$

where $<>_{S U_{q}(2)}$ is the invariant "Haar" measure on $S U_{q}(2)[9]$. This integral is obviously left- and right- invariant under the coaction of $S O_{q}(3)$ and unique as such. The normalization is choosen to give the correct classical limit. Classically, $d \omega^{1}=i / 2 d \Omega$.

Therefore the deformed monople charge is obtained as in the classical case

$$
\frac{1}{2 \pi i} \int_{S_{q}^{2}} F=\frac{q^{2 n}}{2 \pi i} \int_{S_{q}^{2}}[n]_{q^{-2}} d \omega^{1}=q^{2 n}[n]_{q^{-2}} .
$$

This is actually gauge - invariant in the sense that it does not depend on the trivialization chosen, but this appears to be the case only for our particular connection.

\section{Acknowledgements}

We wish to express our gratitude to Prof. Bruno Zumino for many useful discussions, encouragement and support, in particular for drawing our attention to this problem. We also thank Arne Schirrmacher for suggestions, 
and T. Brzezinski for sending us [2]. This work was supported in part by the Director, Office of Energy Research, Office of High Energy and Nuclear Physics, Division of High Energy Physics of the U.S. Department of Energy under Contract DE-AC03-76SF00098 and in part by the National Science Foundation under grant PHY-90-21139.

\section{References}

[1] T. Brzezinski and S. Majid, "Quantum group gauge theory on quantum spaces" Comm. Math. Phys. 157, 591 (1993)

[2] T. Brzezinski and S. Majid, Erratum for "Quantum group gauge theory on quantum spaces" Comm. Math. Phys. 157, 591 (1993)

[3] M. Pflaum, "Quantum groups on Fibre Bundles". Preprint-Nr. gk-mp9403/1, (1994)

[4] R. Budzynski, W. Kondracki, "Quantum Principal Fiber Bundles: Topological Aspects Preprint hep-th/9401019 (1994)

[5] P.M. Hajac, "Strong connections and $U_{q}(2)$ - Yang - Mills Theory on Quantum Principal Bundles" Dissertation, Berkeley (1994)

[6] P.Podles, "Quantum Spheres".Lett.Math.Phys.,14:193-202,(1987).

[7] M.E.Sweedler, Hopf algebras. Benjamin, (1969).

[8] S.L. Woronowicz, "Twisted $S U(2)$ group, an example of a non-commutative differential calculus." Publ. RIMS (Kyoto),23:117$181,(1987)$. 
[9] S.L. Woronowicz, "Compact Matrix Pseudogroups". Comm. Math. Phys. 111, 613 (1987)

[10] S.L. Woronowicz, "Differential Calculus on Compact Matrix Pseudogroups". Comm. Math. Phys. 122, 125 (1989) 